\title{
CERVICO-VAGINAL SWABS TO PREDICT PROM: A CASE STUDY
}

\author{
B. G. Boricha, Shaifali Patil, Nidhi Kurkal, Rama Borse
}

1. Professor \& HOD. Department of Obstetrics \& Gynaecology, MGM Medical College, \& Hospital. Kamothe.

2. Associate Professor. Department of Obstetrics \& Gynaecology, MGM Medical College, \& Hospital. Kamothe.

3. Registrar . Department of Obstetrics \& Gynaecology, MGM Medical College, \& Hospital. Kamothe.

4. Registrar. Department of Obstetrics \& Gynaecology, MGM Medical College, \& Hospital. Kamothe.

\section{CORRESPONDING AUTHOR:}

Dr. Nidhi Kurkal,

802, Fortune Heights,

L.J. Road, Opp. Vijay Sales,

Mahim, Mumbai - 400016.

E-mail: jusgoaway@gmail.com

ABSTRACT: BACKGROUND: The presence of lower genital tract infections poses a threat to the health of a woman. During pregnancy, these infections may also have a direct effect on the fetus or may indirectly predispose to fetal damage secondary to premature labour or premature rupture of membranes. Early detection is thus important because maternal physiological alterations and the presence of a fetus may hamper the diagnosis and the management of such infections. Antepartum treatment of lower genital tract infections or bacterial colonization has been found to reduce the incidence of preterm births. OBJECTIVES: To study the impact of education and socio-economic background, to assess diagnostic efficacy in pregnancy, to study the effect of intervention on the outcome of pregnancy and finally, to study the appropriate trimester for taking a cervicovaginal swab in pregnant women. MATERIALS AND METHODS Our case study was a prospective study involving 100 pregnant women following up in the outpatient department of a community hospital from 2008-2010 with respect to their cervicovaginal microbial growth in each trimester. Patients were divided into culture positive and negative groups and treated accordingly RESULTS - In our study, we found that streptococcus, candida and staphylococcus were the most commonly prevalent among all the microorganisms isolated. Out of 100 women, 27 were swab positive in the $1^{\text {st }}$ trimester with an increasing trend of $1 \%$ in swab positivity in subsequent trimesters. Among swab positive cases, $51.85 \%\left(1^{\text {st }}\right), 50 \%\left(2^{\text {nd }}\right)$ and $51.72 \%\left(3^{\text {rd }}\right)$ landed up in preterm labour with or without PROM.

CONCLUSION - There was a very high positive correlation between the incidence of a positive swab culture and preterm events. We found that the best time for a cervicovaginal swab was during the $3^{\text {rd }}$ trimester. Our study had a high negative predictive value i.e. absence of growth were best in predicting that cases would not go in to preterm labour

INTRODUCTION: Preterm labour is defined as the presence of uterine contractions of sufficient frequency and intensity to effect progressive effacement and dilation of the cervix prior to term gestation (between 20-37 weeks). Preterm births occur in approximately $12 \%$ of pregnancies and is the leading cause of neonatal mortality today ${ }^{(1,2)}$. The exact mechanism of preterm labour is yet unknown but is postulated to be commonly due to decidual haemorrhage (abruption, mechanical factors such as uterine over distension as in multiple gestation or polyhydramnios), cervical incompetence (trauma/cone biopsy), cervical inflammation (due to vaginosis/ trichomonas), uterine distortion (Mullerian anomalies, fibroid), maternal inflammation or fever, hormonal changes or utero-placental insufficiency (hypertension, diabetes, drug abuse etc.) $(1,2)$. 
Intrauterine infections are responsible for $40 \%$ cases of preterm labour that may be overt or subclinical whereas the incidence of positive cultures of amniotic fluid during preterm labour varies from $10-40 \%$, the average being $40 \%$.

Premature rupture of membranes (PROM) is the rupture of membranes prior to the onset of labour beyond 37 completed weeks of gestation. Preterm premature rupture of membranes (PPROM) is the rupture prior to 37 weeks of gestation. Early PROM is mostly due to inflammation and/or infection of the membranes. Clinical factors associated with PPROM are low socio-economic status, low BMI, tobacco use, history of previous preterm labour, urinary tract infections, history of vaginal bleed during pregnancy or cerclage or amniocentesis (3).PROM occurs in $10 \%$ of pregnancies.

The frequency of infections in preterm births is inversely related to the gestational age (4). Endotoxins released by microorganisms and the decidual reaction may stimulate uterine contractions and weaken fetal membranes respectively (5). Bacterial vaginosis has been linked to preterm birth raising the risk by a factor of 1.5 . Extremes of age $(4,7)$, maternal height, weight (8) and decrease in interval of pregnancies increase the incidence of preterm births ${ }^{(9)}$.

Proper intervention with appropriate antibiotics is necessary in those with proven infections. They have two effects i.e. first to increase latency (time till delivery) and secondly to reduce the occurrence of neonatal sepsis, intraventricular haemorrhage and perinatal mortality and chorioamnionitis.

The aim of our study was to study the impact of education, socio-economic status, the effect of intervention in cases with positive cervico-vaginal microbiology on final outcome of pregnancy and to study the appropriate trimester for taking cervico-vaginal swabs in pregnant women.

MATERIALS AND METHODS: Our study was carried out in a community hospital from the year 2008-2010. It was a prospective study involving 100 pregnant women with regular follow up in all three trimesters and they were divided into two groups: culture positive and culture negative according to the swab culture reports. Those with positive reports were treated according to the antibiotic sensitivity test.

The following women were included in the study : -

1. Those with known last menstrual periods (LMP)

2. Presenting from the $1^{\text {st }}$ trimester

3. Having singleton pregnancy

The following women were excluded from the study: -

1. Those with demonstrable maternal cause of preterm labour and PROM

2. Those who refused consent or follow up or defaulters

3. Those with history of liver disease and/or on concurrent intake of hepatotoxic drugs

4. Those with suspected or confirmed fetal congenital malformations

Data collection was done during the OPD visits. Detailed history and thorough clinical examination with special consideration to per speculum examination was done. Cervico-vaginal swabs were taken in each trimester after informed consent along with routine ANC investigations. Patients were treated as per swab culture results. Finally outcomes were compared with respect to the trimester in which they were intervened.

\section{RESULTS:}

Table 1. prevalence of cervico-vaginal infections in various trimesters 
ORIGINAL ARTICLE

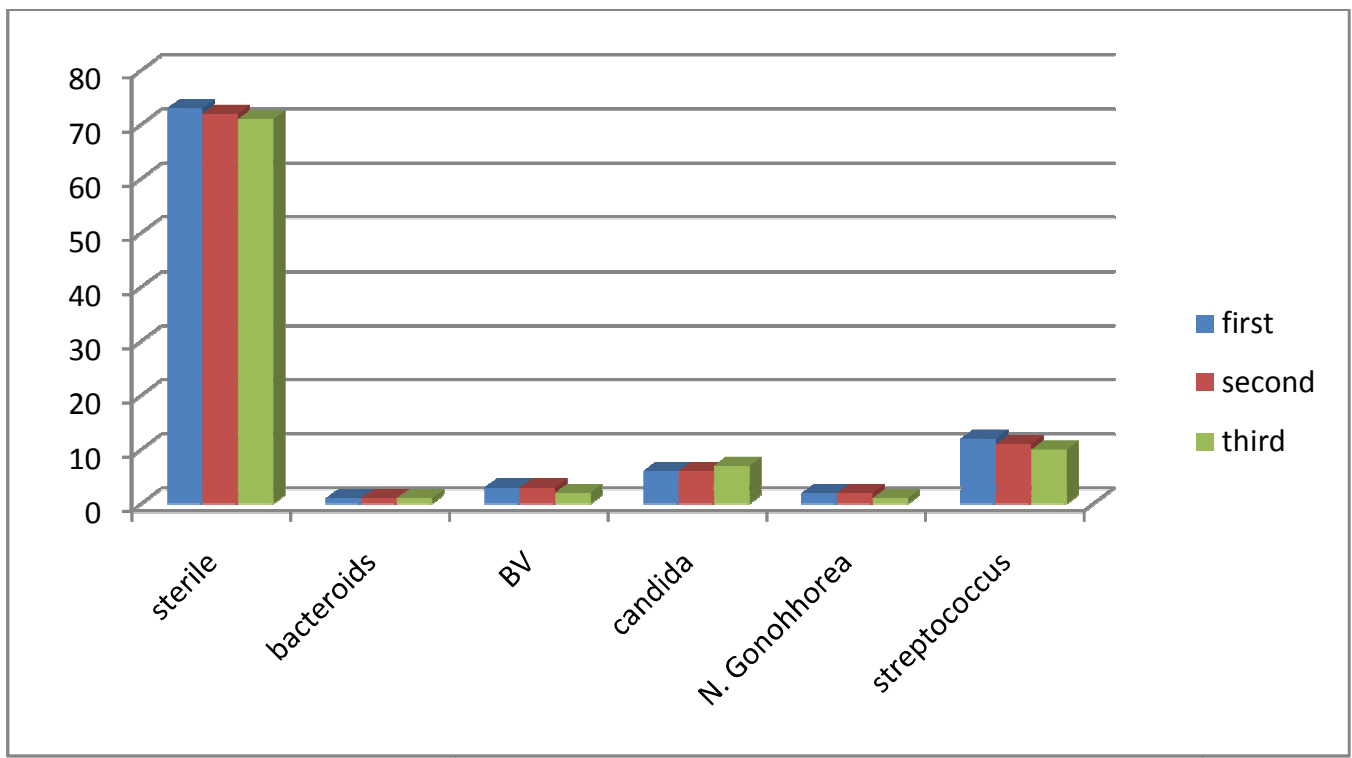

Table 2: Swab results with final outcome in first trimester

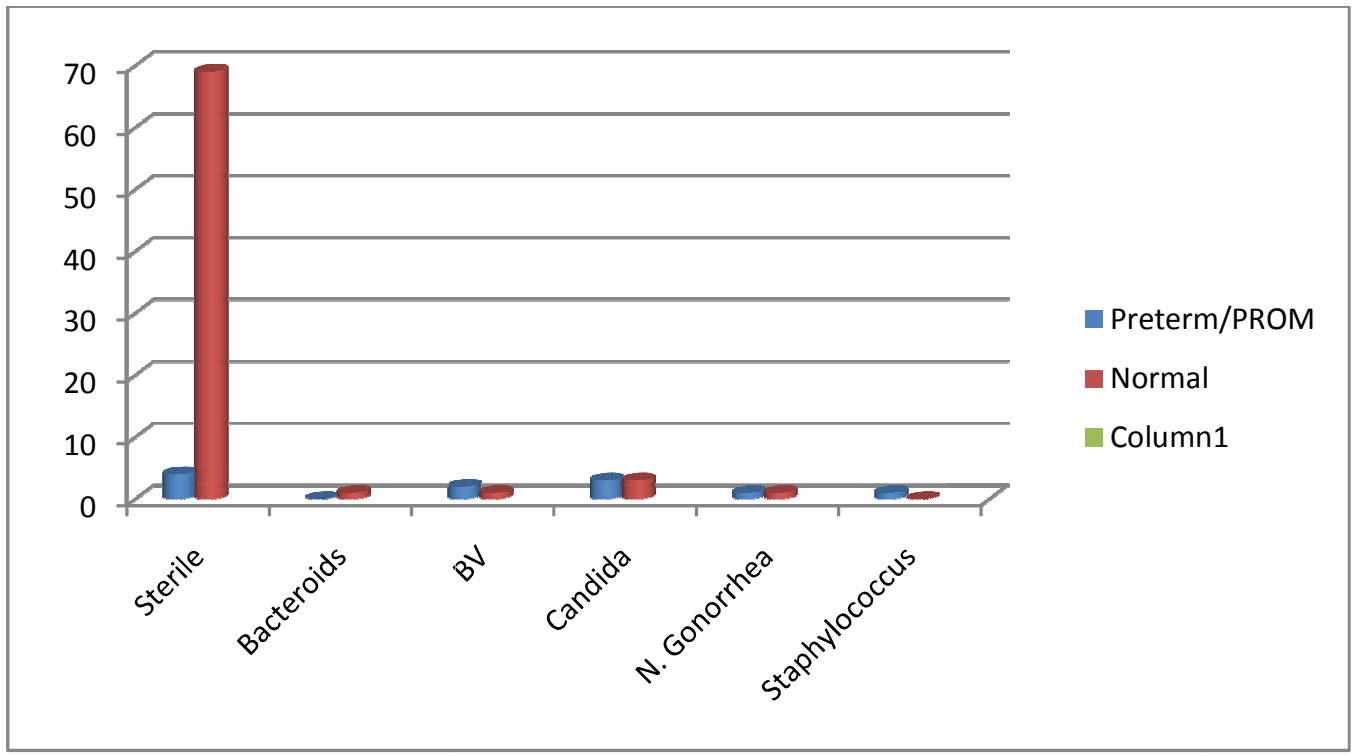

Table 3. Swab results with final outcome in second trimester 


\section{ORIGINAL ARTICLE}

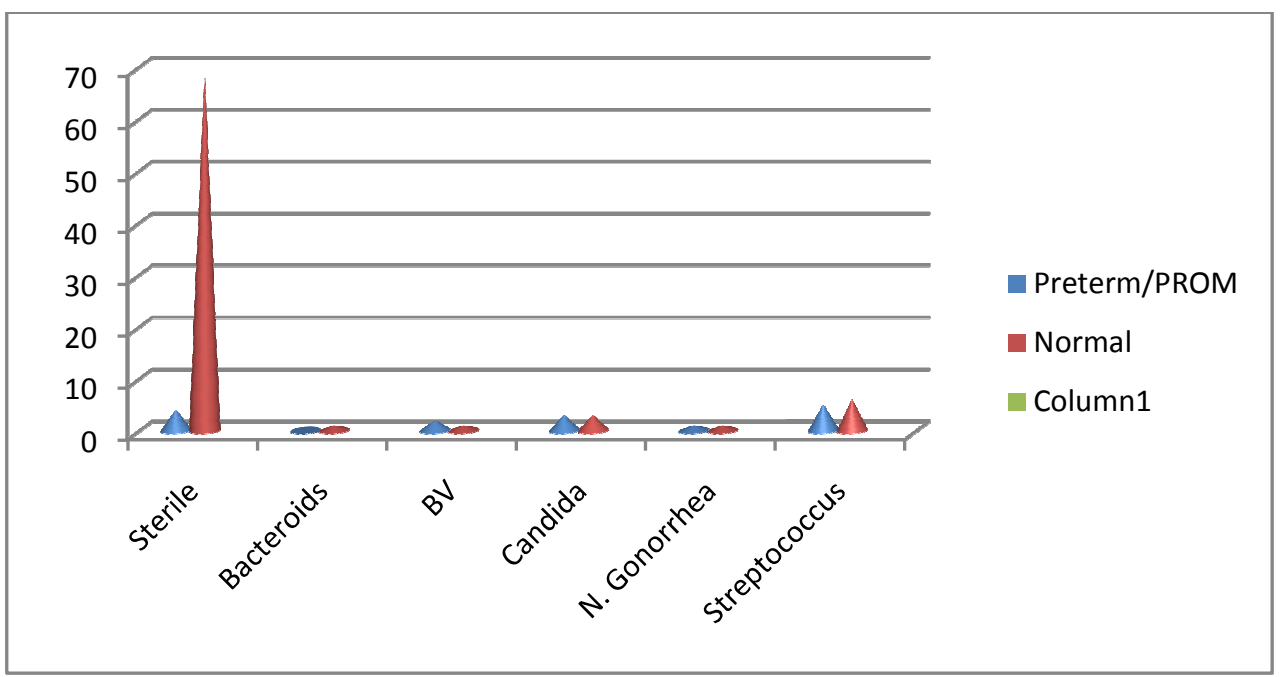

Table 4. Swab results and final outcome in the third trimester

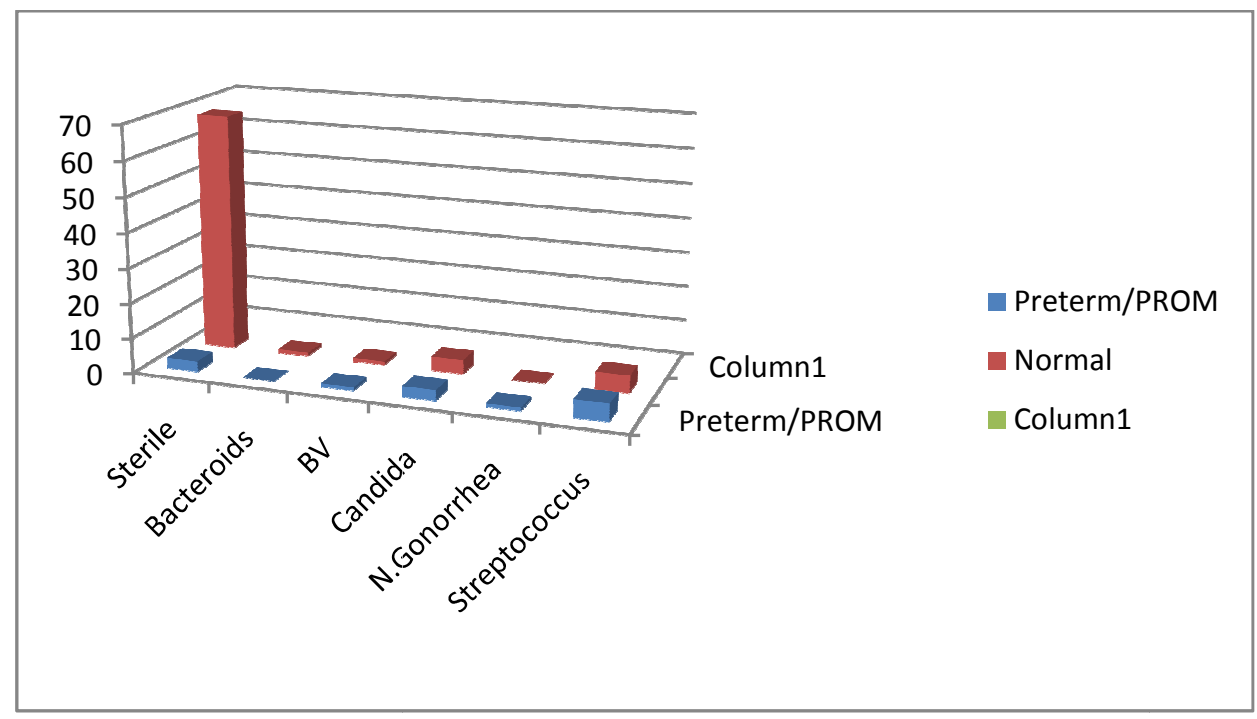

Table 5. Socioeconomic status versus third trimester cervico-vaginal swabs

\begin{tabular}{|l|l|l|l|}
\hline & \multicolumn{3}{l|}{ Socioeconomic Status } \\
\hline Swab Results & Average & Below & Total \\
\hline Positive & 13 & 16 & 29 \\
\hline Negative & 51 & 20 & 71 \\
\hline Total & 64 & 36 & 100 \\
\hline
\end{tabular}

$P$ value -0.011 
DISCUSSION: Of all the swab positive cases in all three trimesters, streptococcus, candida and staphylococcus were the most commonly prevalent micro-organisms among all the microorganisms isolated so far in cervico-vaginal cultures in our study.

The mean age of the pregnant women in our study was 24.51 yrs (min - 18 yrs and max - 38 yrs). It has been found that at the age less than $18 \mathrm{yrs}$ and more than $35 \mathrm{yrs}$, increases the risk of preterm deliveries $(4,7)$.

Out of total 100, 27 women were swab positive in the first trimester (i.e. 27\%) and there was an increasing trend of $1 \%$ in swab positivity in subsequent trimesters as a result of introduction of new infections as pregnancy advanced; new infection rate being less in second trimester than third (i.e. 39.28 versus $48.2 \%$ ).

Of all the organisms, streptococcus (mainly GBS, S. pyogenes), candida and staphylococcus (including coagulase negative) were the most commonly prevalent microorganisms isolated in the cultures in decreasing order of frequency.

Among the swab positive cases in our study, the following percentage landed in preterm labour with or without premature rupture of membranes i.e. $51.58 \%$ (first), 50.0\% (second), $51.72 \%$ (third). The trend of infection rate ultimately causing preterm events in our study is supported by the fact given by Lamont, et al (10) i.e. earlier in pregnancy at which abnormal genital tract colonization is detected, the greater is the risk of late miscarriage and preterm birth.

All culture swab positive cases in our study were treated according to their antibiotic susceptibility and finally the results were analysed. In our study, cure rate when compared with final outcome of pregnancy was found to be best if provided in second trimester amongst all three (i.e. 48.1\%, 50\%, 48.27\% in first, second and third trimester respectively).

Among the 100 women only $10 \%$ were formally educated and $11 \%$ of total sample size in our study had significant past history. However, neither of the two parameters had any statistical significance on the swab culture results in our study. In a few previous studies by Moreau et al(11) and Philip M. Blumenshine et al(12) mentioned the importance of previous obstetric history as well as the impact of maternal as well as paternal education as a risk factor for preterm labour. The exact mechanism is unclear but it is postulated that it could be due to an unfavourable uterine environment in the former and the lack of awareness and improper nutrition in the latter.

Our study did have a significant $\mathrm{P}$ value with respect to socioeconomic status and swab positivity in the third trimester. Out of the 36 women below poverty line (measured by the Kuppuswamy scale), 16 were swab positive i.e.44.44\% ( as compared to the 13 in the remaining 64 women i.e. $20.31 \%$ ) i.e. $55.17 \%$ of the swab positive women were below poverty line.

$48.1 \%, 82.1 \%$ and $65.5 \%$ women were symptomatic in the first, second and third trimesters respectively and $77.7 \%, 75 \%$ and $72.4 \%$ had demonstrable signs in the respective trimester. Interestingly, both symptoms and signs were significant except in the early trimester. In our study, vaginal discharge in the first trimester was poorly related with swab positivity unless patient complained of persistence of symptoms in the subsequent trimesters.

All culture swab positive women were treated according to their antibiotic susceptibility and results were analyzed. Prior studies have revealed that antibiotic treatment results in significantly greater prolongation of pregnancy as compared with placebo in small studies(13) but negative results in studies involving a large number of subjects(14). In our study, the cure rate compared to the final outcome was best when provided in the second trimester. Shennan $\mathrm{AH}$ et al(15) has stated that unnecessary use of antibiotics with no objective evidence of infection 
in spurious preterm labour at late gestations may do more harm than good. Very little difference in the cure rates in subsequent trimesters was seen in our study since intervention had been given to all cases.

Cervicovaginal swab culture has been found to be the most important diagnostic tool to isolate the causative microorganism. Even other studies (16) have revealed its significance in predicting PROM, PPROM and preterm delivery. In fact when compared to a wet mount and cytology methods, it ranks at the top as stated in the International Journal of Tropical Medicine(17-147). Proper culture techniques have been considered the gold standard technique while subjection the selected positive cases to other more accurate tests like real time polymerase chain reaction and optical immunoassay (18-148). In our study, the sensitivity of this method was $77.77 \%$ in the first and second trimester and $83.33 \%$ in the third trimester. The specificity was $84.14 \%$ in the first trimester and $82.92 \%$ in the second and third trimester. The negative predictive value ranged from $94-96 \%$. Thus it is safe to say that culture techniques were the best to predict cases that were not going to land up in preterm labour or premature rupture of membranes.

CONCLUSION: Prevalence of cervico-vaginal infections in our study was 27\% (first trimester), $28 \%$ (second trimester), 29\% ( third trimester) during the pregnancy. Streptococcus, Candida and Staphylococcus were the leading micro-organisms in various trimesters.

The mean age at pregnancy was 24.5 years. The ratio of the uneducated versus the educated was 9:1 and it did not affect swab culture results significantly. Amongst all the parameters, socio-economic status proved to be influencing swab culture with a significant $\mathrm{P}$ value of 0.011 .

Persistent vaginal discharge (with or without itching) in subsequent trimesters and bleeding per vaginum were the two most common clinical presentation in women who had positive swab cultures.

The Diagnostic efficacy of cervico-vaginal swabs was $80 \% \pm 3 \%$ in terms of sensitivity and $83 \% \pm 1 \%$ in terms of specificity. Absence of growth on the cervicovaginal swabs were the best to predict cases that would not have preterm events as proved by the negative predictive value.

Medical intervention was helpful in reducing the preterm events as demonstrable by conversion of culture positive cases to negative cases in our study. Though a pregnant woman is prone for new infections as pregnancy advances but they need to be treated as soon as infection is diagnosed. So, this study concludes that a pregnant woman should be treated with antibiotics depending on the culture sensitivity results.

Third trimester was found to be best time for taking a cervico-vaginal swab in a pregnant woman. However swab may be taken at any time if a pregnant woman complaint of progressively increasing vaginal discharge or at any time she experiences bleeding per vagina in the present pregnancy, as this was found to be statistically significant in our study.

\section{REFERENCES:}

1. American college of obstetrics and gynecologists. ACOG Practice Bulletin. Assessment of risk factors for preterm birth. Clinical management guidelines for obstetricsgynecologists. Number 31, October 2001. (Replaces Technical Bulletin number 206, June 1995; Committee Opinion number 172, May 1996; Committee opinion number 187, September 1997; Committee opinion number 198, February 1998; Committee opinion number 251, January 2001) obstet Gynecol. Oct 2001;98(4):709-16. 
2. ACOG practice bulletin. Management of preterm labour. Number 43, May 2003. Int J Gynecol Obstet. Jul 2003;82(1):127-35.

3. Mercer B, Milluzzi C, Collin M. Periviable birth at 20 to 26 weks of gestation: proximate causes, previous obstetric history and recurrence risk. Am J Obstet Gynecol. Sep 2005; 193 (3 pt 2):1175-80.

4. Goldenburg RL, Culhane JF, Iams JD, Romero R (2008). Epidemiology and causes of preterm birth". The Lancet 371:75-84. Doi: 10.1016/S0140-6736(08)60074-4.

5. Goldenberg RL, Hauth JC, Andrews WW (2000). "intrauterine infection and preterm delivery". New England Journal of Medicine. Doi:10.1056/NEJM200005183422007.342:1500-1507. PMID 10816189.

6. Hiller SL, Nugent RP, Eschenbach DA, et al. (1995). "Association between bacterial vaginosis and preterm dlivery of a low-birth weight infant. The vaginal infection and prematurity study group". New England Journal of Medicine 333.1737-1742. doi: 10.1056/NEJM 199512283332604.

7. Martius JA, Steck T., Oehler MK, Wulf KH (1988). "Risk factors associated with preterm $(<37+0$ weeeks $)$ and early preterm birth $(<32+0$ weeks $)$ : univariate and multivariate analysis of 106345 singleton births from the 1994 statewide perinatal survey of Bavaria"; European Journal of Obstetrics and Gynaecology and Reproductive Biology 80 (2): 183-189. Doi:10.1016/S0301-2115(98)00130-4

8. Merck. "Risk factors present before pregnancy". Merck Manual Home Edition. Merck Sharp \& Dohme

9. Smith GC, Pell JP, Dobbie R (2003). "Interpregnancy interval and risk of preterm birth and neonatal death: retrospective cohort study". British Medical Journal 327: 313. Doi: 10.1136/bmj.327.7410.313

10. H. Honest, Sushma, Khalid S. Khan PEDIATRICS Vol. 117 No.4 April 2006, pp. 1055-1066 (doi: 10.1542/peds.2005-1114)

11. Moreau, Caroline (2005). "Previous induced abortions and the risk of very preterm delivery: Results of the EPIPHAGE study". Obstetrical and Gynaecological Survey 60: 627-628.

12. Patricia Abreu Pinheiro de Lemos, International Journal of Tropical Medicine 4 (2); 76 81,2009

13. Mc Gregor JA, French JI, Seo K. "Adjunctive clindamycin therapy for preterm labor: results of a double blinded placebo controlled trial". Am J Obstet Gynecol 1991; 165 : 867-75

14. Kenyon SL, Taylor DJ, Tarnow Mordi W. "Broad spectrum antibiotics for spontaneous preterm labour: ORACLE II randomized trial". Lancet $2001: 357: 989-94$

15. Shennan AH, Crawshaw S., Briley A., Hawken J., Seed P., Jones G, et al. "A randomized controlled trial of metronidazole for the prevention of preterm birth in women positive for cervico-vaginal fetal fibronectin"; BJOG 2006; 113:65-74, doi: 10.1111/j. 14710528.2005.00788

16. Benedetto C., Tibaldi C., Marozio L., Marini S., Masuelli G., Sozzani P., Latino MA, Pelissetto S.J.,Fetal Neonatal Medicine 2004 Nov 16; Suppl 2:9-12 